\title{
Interferometría holográfica en odontología. Revisión de literatura
}

\section{${ }^{1}$ Docente del Area de Prótesis. Departamento de} Tomie Nakakuki de Campos Odontología, Universidad Federal de Sergipe. Brasil. ${ }^{2}$ Magíster en Prótesis. Facultad de Odontología, Universidad de Sao Paulo. Brasil.

${ }^{3}$ Magíster en Física. Instituto de Física. Universidad de Sao Paulo. Brasil.

${ }^{4}$ Docente del Instituto de Física, Universidad de Sao Paulo. Brasil.

5 Docente del del Departamento de Prótesis

Universidad de Sao Paulo. Brasil.

\section{Correspondencia}

José Chorres

Departamento de Prótese. Faculdade de Odontología. Universidade de São Paulo.

Av. Prof. Lineu Prestes 2227. Cidade Universitária.

CEP 05508-900. Butantá. São Paulo. SP. Brasil.

Teléfono: 00551130917888

e-mail : jecrlalo@yahoo.com
Chorres J, Uono C, Batista LR, Muramatsu M, Campos TN. Interferometría holográfica en odontología. Revisión de literatura. Rev Estomatol Herediana 2005; 15 (2) : 171 - 177.

\section{RESUMEN}

El objetivo de este artículo fue describir las aplicaciones de la interferometría holográfica de doble exposición en la odontología. Esta técnica nos permite realizar análisis de estructuras vivas o inertes, con la gran ventaja de preservar los cuerpos estudiados, siendo capaz de detectar micro-dislocamientos de las superficies de las estructuras estudiadas. Será realizada también una pequeña descripción de los principios del método holográfico.

\section{Palabras clave: INTERFEROMETRÍA / HOLOGRAFÍA}

\section{Holographic interferometry in dentistry. Review of the literature} ABSTRACT

The aim of this paper was to describe the applications of double-exposure holographic interferometry in dentistry. This technique allows for in vivo or in vitro analysis, with the great advantage of preserving the studied and detecting of superficial micro-movements of the structures studied. A small description of the principles of the holographic method is also included.

Keywords: INTERFEROMETRY / HOLOGRAPHY

\section{Introducción}

Tanto la medicina como la odontología han desarrollado su ciencia mediante el uso de diversos recursos tecnológicos. Esta simbiosis, permite la formación de grupos de investigación multidisciplinares, donde participan diversos científicos que aportan lo mejor de sus especialidades, con la finalidad de comprender los fenómenos observados tanto en sistemas vivos, como también para la comprensión del comportamiento de los materiales usados en biotecnología. Esta ultima, incluye por ejemplo el estudio del comportamiento desde una simple amalgama dentro de la estructura dentaria, hasta el análisis del comportamiento del titanio, cuando es usado como material que será colocado dentro de las estructuras óseas, para restablecer las estructuras dentarias perdidas. Diversos métodos han sido desarrollados para evaluar el comportamiento de los materiales restauradores en odontología. Entre los más conocidos podemos encontrar: métodos fotoelásticos, célula de carga y elementos finitos. Otro método poco referido, también ha sido descrito en la literatura y conocido como método holográfico. Dentro de esta técnica holográfica, el método de doble exposición ha sido ampliamente usado en di- versas áreas de la odontología como : ortodoncia, materiales dentarios, prótesis dentaria, oclusión, implantologia, odontología forense y también estudios dirigidos a la comprensión de los fenómenos que rigen el comportamiento biomecánico de las estructuras óseas (1). Una de las ventajas de la técnica holográfica, es la posibilidad de realizar mediciones de los micro-dislocamientos superficiales que ocurren en los cuerpos sometidos a estímulos externos. Aún, se puede realizar evaluaciones directamente en los objetos estudiados, obteniendo así, una observación más real de los fenómenos que ocurren cuando estos objetos son estimulados. El propósito de este artículo es describir las investigaciones que se han desarrollado en el área de odontología y biomecánica ósea usando como método de evaluación a la interferometría holográfica de doble exposición y realizar una breve descripción de esta técnica.

\section{Principios de la holografía}

Para mejorar el entendimiento de los fenómenos holográficos iniciaremos diciendo que una fotografía puede ser comparada con una pintura y un holograma a una escultura. En este ejemplo, en la observación de la pintura sólo se puede visualizar la imagen en dos di- mensiones, en el caso de la escultura, existe nociones de profundidad y se puede observar todos sus contornos, ofreciéndo mayor información sobre el objeto visualizado. Tanto la fotografía como la holografía usan películas o láminas fotográficas, pero la diferencia está en el modo en que la imagen es reproducida. La naturaleza de la imagen óptica (luz), producida por la lente de una cámara, puede ser descrita usando los principios de geometría simples o modelos de rayos. Sin embargo, la imagen holográfica no puede ser descrita por el modelo de rayos y su existencia depende de la difracción e interferencia, que son fenómenos ondulatorios (2). La holografía, un proceso de grabación y proyección de imágenes, que permite la reconstrucción de una escena en tres dimensiones, fue inventada por Gabor en 1948 (3). Para tal fin, una luz monocromática, coherente, paralela e intensa es necesaria: la luz láser. Cada parte del holograma actúa como una lente o un ojo y registra todo lo observado de un objeto, desde un determinado punto de vista. Este registro da el efecto tridimensional cuando observamos un holograma. Inclusive, esta capacidad de observar un objeto en todas sus dimensiones ha sido denominada "paralaxe" y sólo puede ser observada en hologramas 
que han sido producidos con varios puntos de observación del objeto. El holograma consiste en una placa de vidrio o película cubierta con una emulsión fotográfica de altísima resolución (30005000 líneas $/ \mathrm{mm}$ ) y alta sensibilidad (las películas fotográficas convencionales tienen de 100 a 400 líneas $/ \mathrm{mm}$ ). Si este holograma, es expuesto dos veces (doble exposición) y ha ocurrido alguna alteración en el objeto de estudio, entre el tiempo de ambas exposiciones, franjas claras y oscuras aparecerán sobre la imagen capturada en el holograma (4).

Estas franjas son causadas por las diferencias de amplitud y fase de la luz láser que incidió las superficies no modificadas (1 exposición) y modificadas (2 exposición) del objeto sobre acción del estimulo. Estas franjas sirven para realizar un mapeamiento de las alteraciones superficiales del objeto estudiado. De esta forma, cada franja representa una alteración equivalente a la mitad de la longitud de onda de la luz láser. En el caso de haber utilizado un láser de $\mathrm{He}-\mathrm{Ne}$, significa que deformaciones o micro-movimientos de $0,3 \mu \mathrm{m}$ pueden ser medidos (4).

\section{Revisión de literatura \\ Holografía en odontología}

Las técnicas de interferometría holográfica han sido aplicadas con frecuencia en las ciencias biomédicas. Su capacidad para detectar modificaciones mínimas en las dimensiones físicas o en las posiciones relativas de un sistema, permite el estudio del comportamiento de elementos biológicos o inertes en situaciones estáticas o dinámicas sin provocar destrucción de los modelos de estudio (1). Dentro de las aplicaciones más comunes de la holografía en el campo de la odontología y biomecánica ósea, podemos enumerar: estudios realizados para analizar micro-movimientos, para medición de deformaciones mecánicas, para análisis experimental de tensiones, para determinación del factor de intensificación de tensiones-deformaciones y para la determinación de las propiedades de los materiales (5-7). Así, describimos los trabajos de investigación realizados con la ayuda de la técnica de interferometría holográfica de do- ble exposición en odontología y biomecánica ósea.

\section{Prótesis dentaria}

Wictorin, Bjelkhagen y Abramson en 1972 (8) reportaron por primera vez el uso de la holografía para el estudio de la distribución de las tensiones en odontología. Ellos analizaron la deformación elástica en estructuras metálicas soldadas simulando una prótesis fija de tres elementos.

Wedendal y Bjelkhagen en 1974b (9) estudiaron la deformación elástica de dos sistemas de conexiones asociadas a barras, simulando una prótesis dentaria. El padrón de franjas de interferometría holográfica permitió evaluar las condiciones de cada espécimen sobre aplicación de una carga. Sin embargo, ellos mencionaron que, por tratarse de un estudio in vitro las conclusiones deberían ser analizados con cuidado, tendiendo en cuenta que en los sistemas vivos la movilidad dentaria ocurre por la presencia del ligamento periodontal, permitiendo un movimiento diferente de la prótesis dentaria y que estos factores deberían ser estudiados por métodos específicos. En el mismo año Wedendal y Bjelkhagen $1974 \mathrm{c}$ (10) desarrollaron una técnica holográfica que posibilitó la medición dinámica de dientes humanos y de prótesis dentarias. Ellos demostraron que una prótesis dentaria interferente, causó intrusión y rotación del diente antagonista y que esta se comportó como un mono-bloque cuando sometida a carga.

Young y Altschuler en 1974 (11) mencionaron que la técnica holográfica fue capaz de analizar los diferentes tipos de planeamientos y diseños de las prótesis parciales removibles. Esto fue posible mediante el análisis de la distribución de las tensiones producidas por la aplicación de una fuerza oclusal sobre las prótesis.

Young y Altschuler en 1977 (12) presentaron la primera revisión de literatura sobre la técnica y aplicaciones de la interferometría holográfica en odontología. Ellos describieron el proceso de captación y proyección de las imágenes en el holograma, así como mencio- naron las grandes ventajas de la técnica holográfica como : la capacidad de grabar imágenes tridimensionales de objetos e inclusive la posibilidad de realizar mediciones precisas en los cuerpos estudiados.

Las prótesis totales fueron estudiadas, in vitro e in vivo, por Dirtoft en 1980 (13) y Dirtoft, Jansson y Abramson en 1985 (14). Estos autores mencionaron que el análisis de las deformaciones encontradas podría ayudar a la comprensión de los problemas relatados por muchos pacientes que desgastan sus prótesis.

Azizov, Bakhtin y Polukhina en 1985 (15) analizaron la distribución de las deformaciones en una prótesis parcial fija. Ellos observaron que las deformaciones fueron concentradas en las zonas correspondientes a los conectores de la prótesis. Aún mencionaron que, dependiendo del punto de aplicación de la carga, el valor de la deformación de la pieza era alterado, pero la zona de deformación (conectores) se mantenía constante.

Wesson, Goldstein y Schulman en 1986 (16) estudiaron la distribución de las franjas de interferometría holográfica en prótesis parciales fijas después de la aplicación de carga oclusal. En otra investigación evaluaron la distribución de las tensiones en prótesis parciales fijas de tres elementos sobre aplicación de 300 libras de carga sobre el póntico. Concluyeron que la presencia o ausencia del punto de soldaje no influenció el comportamiento de la estructura evaluada. También afirmaron que los padrones de franjas observados en el área del póntico de las prótesis, fueron interpretados como flexión y distribución no uniforme (17).

van Straten, Hitge, Kalk y Schenk en 1991 (18) realizaron un estudio para evaluar la distorsión de resinas usadas como material para confección de prótesis totales. Ellos usaron un disco y una prótesis total de resina y concluyeron que ambos objetos mostraron padrones típicos de deformación, dependiendo del tiempo de realización del test.

Golstein, Wesson, Schweitzer y Cutler en 1992 (19) evaluaron el com- 
portamiento de una prótesis parcial fija de 4 elementos. Después de la aplicación de 200 libras de carga sobre el póntico, ellos observaron la falla del punto de soldaje localizado entre los pónticos. Al analizar los diferentes tipos de padrones de franjas, observaron tensiones de compresión, flexión y compleja deformación.

La aplicación de carga sobre una extensión distal de una prótesis parcial removible fue realizada por Pezzoli, Appendino, Calcagno, Velasco y Modica en 1993 (20). Las prótesis fueron confeccionadas sobre una mandíbula seca humana en la cual fueron extraídos los dientes y recolocados en sus alvéolos con un material que simulaba el ligamento periodontal. Un elastómero de poliéter que simulaba la mucosa oral también fue usado. Padrones de interferencia de franjas fueron observados tanto en la extensión de la resina así como en la superficie del hueso mandibular.

Suliman, Boyer y Lakes en 1993 (21) realizaron mediciones de la deformación de cúspides de dientes restaurados con diferentes resinas. Fueron realizados preparaciones tipo MOD (mesio-oclusodistal) y los efectos del tipo de resina, tamaño de cavidad, así como las condiciones de hidratación fueron evaluados. Concluyeron que dislocamientos menores ocurrieron en las cavidades pequeñas cuando comparadas con las cavidades mayores. También mencionaron que dientes hidratados tuvieron un dislocamiento menor cuando comparados a los dientes no hidratados.

La adaptación marginal de coronas metálicas fue estudiado por Chen, Chang y Wu en 1995 (22). Ellos observaron el efecto de la aplicación de una carga de $25 \mathrm{~N}$ en la fosa central de cada corona protésica. Así, dislocamientos de orden de 6-10 $\mu \mathrm{m}$ pudieron ser observados. Finalmente una abertura marginal de $0,2 \mu \mathrm{m}$ fue observada en todos los casos de aplicación de carga.

\section{Oclusión}

Wedendal y Bjelkhagen en 1974a (23) realizaron un test en modelo de yeso montado en articulador, con el objetivo de establecer criterios que permitiesen realizar la evaluación del dislocamiento de las estructuras dentarias in vivo. Ellos fueron capaces de determinar con exactitud el dislocamiento de las estructuras dentarias evaluadas en el modelo de estudio.

Sanchez del Campo, Bermejo Fenoll, Panchon Ruiz y Jornet Carrillo en 1983 (24) estudiaron las deformaciones del maxilar, mandíbula y dientes humanos al ser sometidos a carga estática, sobre un modelo articulado extraído de un cadáver. Ellos concluyeron que tanto el maxilar como la mandíbula presentaron padrones de interferencia diferentes que sugieren la acción de fibras supracrestales del ligamento alveolar.

La técnica holográfica también ha sido utilizada para el estudio de la dinámica mandibular en modelos de estudio. Reinhardt, Reinhardt y Wenke en 1985 (25) estudiaron la oclusión dentaria en modelos de estudio de resina montados en articulador. Ellos concluyeron que evaluaciones cuantitativas y tridimensionales de las condiciones oclusales pueden ser realizadas con esta técnica. Dislocamientos de cuerpo entero así como alteraciones en la superficie de las estructuras analizadas pueden ser determinadas con gran precisión y sin interferencias.

Batista, Muramatsu, Mori, Campos, Nakao y Secco en 1999 (26) presentaron una investigación que analizaba la distribución de las tensiones en un diente con el método de la interferometría holográfica. El diente fue fijado en una base de resina y fueron realizados hologramas, variando la carga aplicada.

\section{Materiales de impresión}

El análisis de la viscosidad y polimerización de materiales de impresión fue también realizado con la técnica de interferometría holográfica de doble exposición. La estabilidad dimensional fue estudiada por Blandin, Duron y Soulet en 1977 (27), por Senda, Yoshino, Saito, Watanabe, Hara y Tawata en 1979 (28) y por Spajer y Goedgebuer en 1979 (29). Las alteraciones dimensionales de elastómeros fueron investigados por Minchan, Thurgate y Lewis en 1981 (30). El efecto de las cubetas individuales en la deformación de la impresión fue evaluado por Yoshino, Kanamari, Hashimoto et al. en 1985 (31).

\section{Ortodoncia}

La ortodoncia es la especialidad de la odontología que más ha usado la técnica holográfica tanto para grabar imágenes de los modelos dentales en hologramas, así como para la evaluación de la distribución de las tensiones en las estructuras cráneo-faciales.

Una de las grandes ventajas de la técnica holográfica se refiere a la capacidad de grabar imágenes en tres dimensiones y realizar mediciones precisas del objeto en estudio. Esta técnica es importante, pues el espacio ocupado por los modelos de estudio puede ser disminuido notablemente con el uso de películas holográficas (32-38).

Estudios relacionados al dislocamiento de incisivos han sido publicados por Burstone y Pryputniewicz en 1980 (39), por Pryputniewiczy Bowley en 1978 (40) y por Pryputniewicz y Burstone en 1979 (41). El propósito de estas investigaciones fue realizar mediciones más precisas con el objetivo de determinar las perdidas óseas en la enfermedad periodontal, trauma de oclusión y evaluar los efectos de las fuerzas ortodóncicas y protésicas en el macizo cráneo-facial.

Los primeros estudios aplicando la interferometría holográfica en la deformación del cráneo durante los procesos da masticación, fueron realizados por Fuchs y Schott en 1973a,b (42) (43). Hewitt en 1977 (44) aplicó fuerzas ortodóncicas en dientes maxilares y mandibulares en cráneo macerado de monos. Concluyeron que la distribución del stress no fue restringida al complejo dento-alveolar y sí para la totalidad de la superficie cráneo-facial. También mencionaron que los padrones de franjas fueron diferentes en el maxilar y mandíbula debido a las diferencias morfológicas entre ambas estructuras.

Kragt y Duterloo en 1982 (45), Kragt, Duterloo y ten Bosch en 1982 (46) y Kragt, ten Bosch y Borsboom en 1979 (47) aplicaron fuerzas ortodóncicas en cráneo macerado humano. Ellos cuantificaron los movimientos en diferentes 
direcciones y también las deformaciones de los huesos en combinación con varias fuerzas de compresión y cizallamiento en las suturas craneales. Investigaciones previas ya habían mostrado estos resultados $(48,49)$.

Duterloo, Kragt y Algra en 1985 (50) realizaron estudios al respecto del comportamiento de las estructuras cráneofaciales cuando era aplicada carga constante sobre estas estructuras. Ellos demostraron que la distribución de las tensiones y dislocamientos óseos dependen de la morfología individual de cada cráneo macerado. También mencionaron como factores importantes para la distribución del padrón de franjas: el punto de aplicación y la magnitud de la fuerza ejercida.

Canut, Dalmases, Gandia y Salvador en 1990 (51) evaluaron la reacción del complejo cráneo-facial durante la aplicación de fuerzas que inducían al prognatismo del maxilar humano. Ellos concluyeron que existe una rotación mayor del complejo dento-maxilar, cuando la tensión es aplicada en el área de los incisivos laterales. Para los autores esto es demostrado con la extrusión de los molares, en este caso específico. Complementando este estudio Lee, Ryu, Park y Rudolph en 1997 (52) concluyeron que fuerzas de dislocamiento anterior en conjunto con fuerzas de expansión del maxilar, ocasionaron una mayor translación del maxilar que cuando aplicada solamente una fuerza de dislocamiento anterior. De esta forma los autores mencionaron que, mediante el control del punto de aplicación y magnitud de la fuerza, la rotación y translación del maxilar puede ser controlada.

Zentner, Sergl y Filippidis en 1996 (53) evaluaron la distribución de las tensiones aplicando fuerza extra-oral en cráneo seco humano. Concluyeron que la distribución de las tensiones no se limitó al punto de aplicación de la fuerza, sino fueron también distribuidas en el complejo cráneo-facial. También mencionaron que la dirección del dislocamiento no sigue estrictamente la dirección de la aplicación de la fuerza.

Maruyama, Batista, Paiva, Muramatsu y Rino Neto en 2000 (54) evaluaron las áreas de tensión provo- cadas por aparato de expansión rápida del maxilar utilizando la técnica de interferometría holográfica. Ellos observaron cambios en la dirección de las franjas a nivel de las suturas que unen los huesos. Mencionaron también que hubo dislocamientos en sentido vertical-superior en la región de los molares y lateralmente en los procesos dentoalveolares y para adelante y para abajo en el maxilar. Esto ocurrió después de la activación del tornillo expansor, que liberó fuerza en la región de los primeros premolares y primeros molares. Estudio similar fue realizado por Braun, Bottrel, Lee, Lunazzi y Legan en 2000 (55) que evaluaron la distribución de las franjas de interferometría sobre las estructuras cráneo-faciales y sugirieron modificaciones del aparato de expansión usado.

\section{Odontología forense}

Oliveira, Mallet, Melani, Antunes, Batista y Muramatsu en 2000 (56) realizaron un estudio donde aplicaron carga en un cráneo seco humano. El objetivo de esta investigación fue evaluar la distribución de las tensiones sobre el complejo cráneo-facial. Ellos observaron que hubo alteración de la distribución de las tensiones sobre el cráneo en la presencia de suturas "abiertas". Concluyeron que este hallazgo es de gran importancia para estudios posteriores que intenten padronizar tests que consigan determinar la edad ósea de cráneos no identificados, mediante el uso de la técnica de interferometría de doble exposición.

\section{Biomecánica ósea}

El uso de la técnica holográfica para aplicación biomédica viene siendo discutido desde comienzos de los años 70 como mencionado por Chernukh, Danilov, Miller y Siniakov en 1976 (57). En esta publicación diversas técnicas holográficas son descritas así como la posible padronización de éstas para aplicación en el área médica.

Hewitt y Orth en 1981 (58) realizaron una evaluación holográfica de las respuestas óseas durante la acción de pequeñas fuerzas. Para esta investigación fueron usados cilindros de hueso frescos, libres de tejidos blandos y mantenidos en ambiente húmedo. Ellos conclu- yeron que la técnica holográfica puede ser usada para evaluar cualitativa y cuantitativamente el padrón de stress observado sobre la superficie del objeto estudiado. También mencionaron que la distribución de las franjas depende de la morfología del objeto en estudio (hueso), de la dirección y magnitud de la fuerza aplicada y que el stress generado sobre la superficie ósea no queda restricto al punto de aplicación de la fuerza.

Iroshnikova, Kudrin, Perk, Polukhina y Timofeeva-Kol'tsova en 1982 (59) analizaron las deformaciones producidas en la articulación témporomandibular cuando es sometida a carga. Concluyeron que es posible investigar las micro-deformaciones de las articulaciones craneales mediante la técnica holográfica de doble exposición.

Ferre, Legoux, Helary et al. en 1985 (60) estudiaron el comportamiento de una mandíbula fresca humana (postmortis) y un bloque de acero. Concluyeron que el comportamiento de ambas estructuras sólo pueden ser consideras similares en el inicio de la carga sobre las mismas condiciones. También mencionaron que el comportamiento mecánico de la mandíbula podría ser comparado con un compósito heterogéneo y que presenta el fenómeno de "histeresis", pues cuando sometido a pequeña carga, sufre alteraciones en su forma y que posteriormente, si la carga persistiese las alteraciones serian neutralizadas en función del tiempo.

Un estudio interesante sobre el stress de huesos del cráneo (modelo animal) durante la masticación fueron realizados por Nygrén, Silvennoinen y Karna en 1992 (61). Ellos realizaron una simulación de actividad uni-lateral del músculo masetero durante la masticación. Ellos encontraron que los efectos de la aplicación de carga pueden ser observados en toda la superficie del cráneo con diversas intensidades dependiendo de la estabilidad del hueso y de las suturas craneales. Áreas de rápidos dislocamientos superficiales fueron observadas en las zonas correspondientes a la fijación del músculo masetero y alrededor de la unión nasofrontal. Ellos concluyeron que esta distribución de fuerza puede ser la respon- 
sable por la variación de las estructuras óseas nasales de esta especie.

Silvennoinen, Nygrén y Karna en 1992 (62)(63) demostraron el uso de la interferometría holográfica como método para evaluar la distribución de las tensiones en mandíbulas fracturadas (modelo animal) intencionalmente y fijadas con placas rígidas. Observaron que las mandíbulas con período de cicatrización de 4 semanas no fueron capaces de transmitir los padrones de franjas cuando era aplicada fuerza en la zona correspondiente a los incisivos. La mandíbula con 8 semanas de cicatrización fue capaz de transmitir el padrón de franjas uniformemente en el hueso mandibular, con o sin la presencia de la placa de fijación.

Los mismos autores en trabajo publicado en 1992 (64), realizaron mediciones holográficas en cráneos macerados y frescos de origen animal. Ellos concluyeron que los huesos frescos son dos veces más elásticos que los huesos macerados cuando sometidos a carga.

Batista, Muramatsu y Campos en 2003 (65) y Campos en 2001 (66) realizaron un análisis de mandíbulas de canes en diferentes estados : frescas (postmortis), maceradas (secas) y fijadas en formol mediante la técnica de interferometría holográfica de doble exposición. Ellos concluyeron que la deformación de las mandíbulas frescas fue menor debido a la presencia del ligamento periodontal, ya que diente y hueso se comportaron de manera diferente. En el caso de las mandíbulas fijadas y maceradas, estas se comportaron como un cuerpo rígido. También las mandíbulas maceradas y fijadas sufrieron mayor torsión durante el dislocamiento que las mandíbulas frescas.

Uono en 2005 (67), estudió la influencia de coronas protésicas de metalocerámica y de resina, cementadas en diente canino (modelo animal), en la transmisión de tensiones para el tejido óseo de mandíbulas frescas (post-mortis) de canes. Una carga de $0,98 \mathrm{~N}$ fue aplicada sobre las coronas, siguiendo el eje axial del diente. Fueron obtenidos 12 hologramas, 6 para cada grupo de muestras. Las coronas metalocerámicas presentaron mayor dislocamiento, con movimiento de intrusión en el alvéolo, en re- lación a las coronas de resina. Las mandíbulas demostraron comportamientos semejantes de deflexión, por lo tanto, no fueron encontradas diferencias estadísticamente significantes en la distribución de las tensiones al hueso con relación a los dos tipos de coronas consideradas, demostrando la influencia del ligamento periodontal.

\section{Implantología}

Dirtoft y Jansson en 1986 (68) estudiaron la deformación de una prótesis sobre implante, sobre la acción de una carga. Ellos observaron que la deformación era compleja, un mixto de deflexión sobre carga de baja intensidad y torsión sobre cargas más elevadas.

Wahl y Lang en 2004 (69) evaluaron la deformación de superficies de implantes de titanio y de coronas protésicas sometidas a carga estática. Verificaron que los implantes de titanio mostraron una distribución de franjas homogéneas (horizontales) entre si y cuando era aplicada la carga sobre las coronas protésicas, éstas mostraron padrones de dislocamiento variado (franjas oblicuas) y formación de espacios entre la plataforma del implante y la base de la prótesis. Con esto concluyeron que la alta precisión de los componentes para rehabilitación con implantes es afectada en gran parte por las deformaciones dinámicas cuando aplicada carga funcional.

Finalmente Chorres en 2005 (70), estudió la distribución de las tensiones a través de prótesis fijas implanto-soportadas y dento-implanto-soportadas en mandíbulas de canis familiaris mediante el método de interferometría holográfica de doble exposición. Tres canes fueron seleccionados y colocados tres implantes en cada uno de los animales (mandíbula derecha : un implante y en la mandíbula izquierda dos implantes). Después de dos meses de espera, los dos tipos de prótesis fueron fijados sobre los implantes y los animales fueron sacrificados. A seguir, las mandíbulas fueron extraídas y fijadas en dispositivos especialmente diseñados para este fin (66). Todo el sistema fue colocado sobre una mesa holográfica y carga estática fue aplicada sobre tres posiciones previamente establecidas en las próte- sis (sobre el pilar anterior, punto medio de la barra y pilar posterior). 18 hologramas fueron obtenidos mediante la técnica holográfica. El análisis de los hologramas reveló que de forma general, las mandíbulas con prótesis dento-implanto-soportadas sufrieron mayor tensión que las mandíbulas con prótesis implanto-soportadas. En relación a las prótesis, las implanto-soportadas fueron las que mejor distribuyeron los esfuerzos cuando comparadas con las prótesis dento-implanto-soportadas.

\section{Conclusiones}

- La técnica holográfica es un método eficaz para el análisis y medición de las alteraciones superficiales en sistemas inertes y biológicos.

- En los últimos años puede ser observada una tendencia mayor al estudio y comprensión de los fenómenos biomecánicos que ocurren cuando sistemas inertes interaccionan con el tejido óseo.

\section{Referencias bibliográficas}

1. Chorres JE. Análise da distribuição das tensões através de prótese fixa implanto-suportada e dento-implanto-suportada em hemi-mandíbulas frescas de "canis familiaris" mediante o método de interferometría holográfica de dupla exposição [Tesis para obtener el grado de doctor en Prótesis Dentaria] Sao Paulo: Facultad de Odontologia. USP; 2005.

2. Batista LR. Interferometría holográfica aplicada à odontologia [Disertación para obtener el título de magíster en ciencias] Sao Paulo: Universidad de Sao Paulo, 2003.

3. Gabor D. A new microscopic principles. Nature 1948;161(4098):777-8.

4. Dirtoft BI. Dental holographiy-earlier investigations and prospective possibilities. Adv Dent Res 1987;1(1): 8-13.

5. Zentner A, Sergl HG, Filippidis G. A holographic study of variations in bone deformations resulting from different headgear forces in a macerated human skull. Angle Orthod 1996;66(6):463-72.

6. Pavlin D, Vukicevic D. Mechanical 
reations of facial skeleton to maxillary expansion determined by laser holography. Am J Orthod 1984;85 (6):498-507.

7. Isaacson RJ, Ingram AH. Forces produced by rapid maxillary expansionpart II: Forces present during treatment. Angle Orth 1964;34(4):256-69.

8. Wictorin L, Bjelkhagen H, Abramson N. Holographic investigation of the elastic deformation of defective gold solder joints. Acta Odontol Scand 1972;30(6):659-70.

9. Wedendal PR, Bjelkhagen HI. Holographic interferometry on the elastic deformation of prosthodontic appliances as simulated by bar elements. Acta Odontol Scand 1974b; 32(3):189-99.

10. Wedendal PR, Bjelkhagen HI. Dental holographic interferometry in vivo utilizing a ruby laser system II. Clinical applications. Acta Odontol Scand 1974c;32(5):345-56.

11. Young JM, Altschuler BR. Laser holographic stress analysis of removable partial denture connectors [abstract 895]. J Dent Res 1974; 53:278.

12. Young JM, Altschuler BR. Laser holography in dentistry. J Prosthet Dent 1977;38(2):216-25.

13. Dirtoft BI. Dimensional changes due to inibition in different processed dentures [abstract 21]. J Dent Res 1980;59:1902.

14. Dirtoft BI, Jansson JF, Abramson NH. Using holography for measurement of in vivo deformation in a complete maxillary denture. J Prosthet Dent 1985;54(6):843-6.

15. Azizov MA, Bakhtin VG, Polukhina SP. Use of optical holography for research on the stressed deformation state of dental bridgework made of metal. Stomatologiia 1985;64(6):66-8.

16. Wesson A, Goldstein G, Schulman A. Stress analysis of fixed prosthodontics frameworks utilizing holographic interferometry [abstract 1334]. J Dent Res 1986;65:317.

17. Wesson A, Goldstein GR, Schulman A. Flexion characteristics of fixed partial denture frameworks tested by using elapsed-time holographic interferometry. J Prosthet Dent
1988;60(3):308-10.

18. van Straten RJ, Hitge ML, Kalk W, Schenk J. A study of acrylic resin denture base material distortion using computer-aided holographic interferometry. Int J Prosthodont 1991;4(6):577-85.

19. Golstein GR, Wesson A, Schweitzer K, Cutler B. Flexion characteristics of four-unit fixed partial denture frameworks using holographic interferometry. J Prosthet Dent 1992;67(5):609-13.

20. Pezzoli M, Appendino P, Calcagno L, Celasco M, Modica R. Load transmission evaluation by removable distal-extension partial dentures using holographic interferometry. J Dent 1993;21(5):312-6.

21. Suliman AA, Boyer DB, Lakes RS. Interferometric measurements of cusp deformation of teeth restored with composites. J Dent Res 1993;72 (11):1532-6.

22. Chen TY, Chang GL, Wu SH. Holographic evaluation of the marginal fits of complete crowns loaded at the central fossa. Opt Eng 1995;34 (5):1364-8.

23. Wedendal PR, Bjelkhagen HI. Dental holographic interferometry in vivo utilizing a ruby laser system. I. Introduction and development of methods for precision measurements on the functional dynamics of human teeth and prosthodontic appliances. Acta Odontol Scand 1974a;32(2):131-45.

24. Sanchez del Campo JF, Bermejo Fenoll A, Panchon Ruiz A, Jornet Carrillo V. Dentomaxillary deformation under pressure using holographic interferometry. Rev Esp Estomatol 1983;31(5):345-50.

25. Reinhardt W, Reinhardt R, Wenke L. Holographic interferometry studies as a supplementary possibility to instrumental occlusal analysis. Stomatol DDR 1985;35(1):9-13.

26. Batista LR, Muramatsu M, Mori M, Campos TN, Nakao E, Secco A. Interferometría holográfica aplicada ao estudo da distribuição de tensões em dente submetido a carregamento. In: Anais da16a Reunião Anual da Sociedade Brasileira de Pesquisa
Odontológica; 1999; Águas de São Pedro. São Paulo: Sociedade Brasileira de Pesquisa Odontológicas; 1999. p. 155, Res.B215.

27. Blandin M, Duron C, Soulet H. Study by holographic interferometry of dimensional variability in presicion-moulding materials used in odontology. Proc SPIE 1977;136:130-4.

28. Senda A, Yoshino H, Saito K, Watanabe T, Hara G, Tawata M. Study on the deformation of impression. Preliminary report: Application of the holographic interferometry. Aichi Gakuin Daigaku Shigakkai Shi 1979;17(3):205-15.

29. Spajer M, Goedgebuer JP. Interferometric studies of the dimensional variations of silicone elastomers. Optics Lasers Technol 1979;11:100-2.

30. Minchan W, Thurgate SM, Lewis AJ. Measurement of dimensional stability of elastomeric impression materials by holographic interferometry. Aust Dent J 1981;26(6):395-99.

31. Yoshino H, Kanamari K, Hashimoto M, Tani T, Mizuno T, Kondo M, Senda A. Effect of the tray on the deformation of the impression. A holography interferometric study. Aichi Gakuin Daigaku Shigakkai Shi 1985;23(1):300-7.

32. Buschang PH, Ceen RF, Schroeder JN. Holographic storage of dental casts. J Clin Orthod 1990;24(5):308-11.

33. Harradine N, Suominen R, Stephens C, Hathorn I, Brown I. Holograms as substitutes for orthodontic study casts: a pilot clinical trial. Am J Orthod Dentofacial Orthop 1990;98(2):110-6.

34. Keating PJ, Parker RA, Keane D, Wright L. The holographic storage of study models. Br J Orthod 1984;11(3):119-25.

35. Mallet RG, Oliveira RN, Batista LR, Muramatsu M. Documentação em odontologia: armazenagem de imagem de modelos de estudo usando hologramas. Odontol e Soc 2000;2(1/2):58-61.

36. Martensson B, Ryden H. The 
holodent system, a new technique for measurement and storage of dental casts. Am J Orthod Dentofacial Orthop 1992;102(2):113-9.

37. Rydén H, Bjelkhagen H, Söder PO. Movements of health and periodontically involved teeth measured with laser reflection technique. J Periodontol 1982;53(7):439-45.

38. Schwaninger B, Schmidt RJ, Hurst RVV. Holography in dentistry. J Am Dent Assoc 1977; 95:814-7.

39. Burstone CJ, Pryputniewicz RJ. Holographic determination of centers of rotation produced by orthodontic forces. Am J Orthod 1980;77:396-409.

40. Pryputniewicz RJ, Bowley WW. Techniques of Holographic Displacement Measurement: An Experimental Comparison. App Opt 1978;17:1748-56.

41. Pryputniewicz RJ, Burstone CJ. The effect of time and force magnitude on orthodontic tooth movement. J Dent Res 1979; 58:1754-64.

42. Fuchs P, Schott D. Anwendung der Holographie bei Verformungsmessungen des menschlicher Gesichtsschädels. Dtsch Zahnärztl Z 1973a;28:90.

43. Fuchs P, Schott D. Holografische interferometrie zur Darstellung von Verformungen des menschlichen Gesichtsschadels. Schweiz Monatsschr Zahnmed 1973b;83(12): 1468-82.

44. Hewitt AB. An investigation using holographic interferometry of surface strain in bone induced by orthodontic forces: A preliminary report. Br J Orthod 1977;4:39-41.

45. Kragt G, Duterloo HS. The initial effects of orthopedic forces: A study of alterations in the craniofacial complex of a macerated human skull owing to high-pull headgear traction. Am J Orthod 1982;81:57-64.

46. Kragt G, Duterloo HS, ten Bosch JJ. The initial reaction of a macerated human skull caused by orthodontic cervical traction determined by laser metrology. Am J Orthod. 1982;81(1): 49-56.

47. Kragt G, ten Bosch JJ, Borsboom PC. Measurement of bone displacement in a macerated human skull induced by orthodontic forces; a holographic study. J Biomech 1979;12(12):905-10.

48. Dermaut LR, Beerden LL. The effects of class II elastic force on a dry skull measured by holographic interferometry. Am J Orthod 1981;79(3):296-304.

49. Duterloo HS, Kragt G, Algra AM. Holographic and cephalometric study of the relationship between craniofacial morphology and the initial reactions to high-pull headgear traction. Am J Orthod 1985;88(4): 297-302.

50. Canut JA, Dalmases F, Gandia JL, Salvador R. Effects of maxillary protraction determined by laser metrology. Eur J Orthod 1990;12(3): 340-5.

51. Lee KG, Ryu YK, Park YC, Rudolph DJ. A study of holographic interferometry on the initial reaction of maxillofacial complex during protraction. Am J Orthod Dentofacial Orthop 1997;111(6):623-32.

52. Maruyama NE, Batista LR, Paiva JB, Muramatsu M, Rino Neto J. Avaliação das áreas de tensão promovidas pelo aparelho de expansão rápida da maxila utilizando-se a técnica de interferometría holográfica. RPG 2000; 7:26.

53. Braun S, Bottrel JA, Lee KG, Lunazzi JJ, Legan HL. The biomechanics of rapid maxillary sutural expansion. Am J Orthod Dentofacial Orthop 2000;118(3):257-61.

54. Oliveira RN, Mallet RG, Melani RFH, Antunes JLF, Batista LR, Muramatsu M. Perspectiva do emprego da holografia como recurso auxiliar no estudo da estimativa da idade pela análise da cronologia de fechamento das suturas craniais. Odontol Soc 2000; 2(1/2):8-11.

55. Chernukh AM, Danilov VA, Miller GS, Siniakov VS. Some problems in the use of holography in medicalbiological studies. Med Tekh 1976;(1):30-3.

56. Hewitt AB, Orth D. Holographic investigation of bone response to small forces. Brit J Orthod 1981; 8(1):43-6.

57. Iroshnikova ES, Kudrin AB, Perk ON, Polukhina SP, TimofeevaKol'tsova TP. Use of holographic interferometry in studying the tem- poromandibular joint. Stomatologiia 1982;61(4):46-7.

58. Ferre JC, Legoux R, Helary JL, Albugues F, Le Floc'h C, Bouteyre J, et al. Study of the mandible under static constraints by holographic interferometry. New biomechanical deductions. Anat Clin 1985;7(3):193201.

59. Nygrén K, Silvennoinen R, Karna M. Masticatory stress on cranial bones; a holographic study. Proc SPIE 1992;1647:164-168.

60. Silvennoinen R, Nygrén K, Karna M. Biomechanical testing of isolated bones: a holographic study. Opt Eng 1992a;31(8):1690-4.

61. Silvennoinen R, Nygrén K, Karna M. Holographics nondestrutive testing in bone biomechanics. Proc SPIE 1992c;1647:156-63.

62. Silvennoinen R, Nygrén K, Karna M. Holographic measurements of fresh and dry bone elasticy. Opt Eng 1992b;31(8):1695-7.

63. Batista LR, Muramatsu M, Campos TN. Stress analysis of fresh and macerated dog mandibles. Holographic interferometric double exposition method. Proc SPIE 2003;4829: 1012-13.

64. Campos TN. Análise das tensões em mandíbulas frescas, fixadas e maceradas de cães: método da interferometría holográfica de dupla exposição [Tesis de Libre Docencia]. Sao Paulo: Facultad de Odontologia. USP; 2001.

65. Uono C. Efeito dos diferentes materiais restauradores protéticos na dissipação da carga oclusal. Método da interferometría holográfica [Disertación para obtener el título de Magíster en Prótesis Dentaria]. Sao Paulo: Facultad de Odontologia. USP; 2005.

66. Dirtoft BI, Jansson JF. Holography and deformation analysis of na implant bridge during loading [abstract 448]. J Dent Res 1986;65: 775.

67. Wahl G, Lang H. Deformation at the implant interface to prosthetic superstructure: an interferometric approach. Clin Oral Implants Res 2004;15(2):233-8. 